\title{
THE INEQUALITY OF MILNE AND ITS CONVERSE II
}

\author{
HORST ALZER AND ALEXANDER KOVAČEC
}

Received 15 September 2004; Accepted 19 September 2004

We prove the following let $\alpha, \beta, a>0$, and $b>0$ be real numbers, and let $w_{j}(j=1, \ldots, n$; $n \geq 2)$ be positive real numbers with $w_{1}+\cdots+w_{n}=1$. The inequalities $\alpha \sum_{j=1}^{n} w_{j} /\left(1-p_{j}^{a}\right) \leq$ $\sum_{j=1}^{n} w_{j} /\left(1-p_{j}\right) \sum_{j=1}^{n} w_{j} /\left(1+p_{j}\right) \leq \beta \sum_{j=1}^{n} w_{j} /\left(1-p_{j}^{b}\right)$ hold for all real numbers $p_{j} \in$ $[0,1)(j=1, \ldots, n)$ if and only if $\alpha \leq \min (1, a / 2)$ and $\beta \geq \max \left(1,\left(1-\min _{1 \leq j \leq n} w_{j} / 2\right) b\right)$. Furthermore, we provide a matrix version. The first inequality (with $\alpha=1$ and $a=2$ ) is a discrete counterpart of an integral inequality published by E. A. Milne in 1925.

Copyright @ 2006 Hindawi Publishing Corporation. All rights reserved.

\section{Introduction}

Motivated by an interesting paper of Rao [8], we proved in [1] the following doubleinequality for sums.

Proposition 1.1. Let $w_{j}(j=1, \ldots, n ; n \geq 2)$ be positive real numbers with $w_{1}+\cdots+w_{n}=1$. Then we have for all real numbers $p_{j} \in[0,1)(j=1, \ldots, n)$,

$$
\left(\sum_{j=1}^{n} \frac{w_{j}}{1-p_{j}^{2}}\right)^{c_{1}} \leq \sum_{j=1}^{n} \frac{w_{j}}{1-p_{j}} \sum_{j=1}^{n} \frac{w_{j}}{1+p_{j}} \leq\left(\sum_{j=1}^{n} \frac{w_{j}}{1-p_{j}^{2}}\right)^{c_{2}},
$$

with the best possible exponents

$$
c_{1}=1, \quad c_{2}=2-\min _{1 \leq j \leq n} w_{j}
$$

The left-hand side of (1.1) (with $c_{1}=1$ ) is a discrete version of an integral inequality due to Milne [7]. Rao showed that (1.1) (with $c_{1}=1$ and $c_{2}=2$ ) is valid for all $w_{j}>0$ $(j=1, \ldots, n)$ with $w_{1}+\cdots+w_{n}=1$ and all $p_{j} \in(-1,1)(j=1, \ldots, n)$.

Double-inequality (1.1) admits the following matrix version; see $[1,8]$.

Proposition 1.2. Let $w_{j}(j=1, \ldots, n ; n \geq 2)$ be positive real numbers with $w_{1}+\cdots+$ $w_{n}=1$ and let $I$ be the unit matrix. Then we have for all families of commuting Hermitian 
2 The inequality of Milne and its converse II

matrices $P_{1}, \ldots, P_{n}$ with $0 \leq P_{j}<I(j=1, \ldots, n)$,

$$
\left(\sum_{j=1}^{n} w_{j}\left(I^{2}-P_{j}^{2}\right)^{-1}\right)^{c_{1}} \leq \sum_{j=1}^{n} w_{j}\left(I-P_{j}\right)^{-1} \sum_{j=1}^{n} w_{j}\left(I+P_{j}\right)^{-1} \leq\left(\sum_{j=1}^{n} w_{j}\left(I^{2}-P_{j}^{2}\right)^{-1}\right)^{c_{2}},
$$

with the best possible exponents

$$
c_{1}=1, \quad c_{2}=2-\min _{1 \leq j \leq n} w_{j}
$$

In Section 2 we provide new bounds for $\sum_{j=1}^{n} w_{j} /\left(1-p_{j}\right) \sum_{j=1}^{n} w_{j} /\left(1+p_{j}\right)$, which are closely related to those given in (1.1). It turns out that the new upper bound and the upper bound in (1.1) cannot be compared. And in Section 3 we present a matrix analogue of our discrete double-inequality.

\section{Inequalities for sums}

The following counterpart of Proposition 1.1 holds.

Theorem 2.1. Let $\alpha, \beta, a>0$, and $b>0$ be real numbers. Further, let $w_{j}(j=1, \ldots, n ; n \geq 2)$ be positive real numbers with $w_{1}+\cdots+w_{n}=1$. The inequalities

$$
\alpha \sum_{j=1}^{n} \frac{w_{j}}{1-p_{j}^{a}} \leq \sum_{j=1}^{n} \frac{w_{j}}{1-p_{j}} \sum_{j=1}^{n} \frac{w_{j}}{1+p_{j}} \leq \beta \sum_{j=1}^{n} \frac{w_{j}}{1-p_{j}^{b}}
$$

hold for all real numbers $p_{j} \in[0,1)(j=1, \ldots, n)$ if and only if

$$
\alpha \leq \min (1, a / 2), \quad \beta \geq \max \left(1,\left(1-\min _{1 \leq j \leq n} w_{j} / 2\right) b\right)
$$

Proof. Let $w=\min _{1 \leq j \leq n} w_{j}$ and $c=2 /(2-w)$. First, we suppose that $\beta \geq \max (1, b / c)$. Since

$$
\max (1, b / c) \geq \frac{1-p^{b}}{1-p^{c}} \quad(0 \leq p<1)
$$

we obtain

$$
\beta \sum_{j=1}^{n} \frac{w_{j}}{1-p_{j}^{b}} \geq \sum_{j=1}^{n} \frac{w_{j}}{1-p_{j}^{c}}
$$

To prove the right-hand side of (2.1) we may assume that

$$
0 \leq p_{n} \leq p_{n-1} \leq \cdots \leq p_{1}<1 .
$$


We define

$$
\begin{gathered}
F\left(p_{1}, \ldots, p_{n}\right)=\sum_{j=1}^{n} \frac{w_{j}}{1-p_{j}^{c}}-\sum_{j=1}^{n} \frac{w_{j}}{1-p_{j}} \sum_{j=1}^{n} \frac{w_{j}}{1+p_{j}}, \\
F_{q}(p)=F\left(p, \ldots, p, p_{q+1}, \ldots, p_{n}\right), \quad 1 \leq q \leq n-1, p_{q+1}<p<1 .
\end{gathered}
$$

Differentiation leads to

$$
\frac{\left(1-p^{2}\right)^{2}}{W_{q}} F_{q}^{\prime}(p)=c p^{c-1}\left(\frac{1-p^{2}}{1-p^{c}}\right)^{2}-2 p W_{q}+\sum_{j=q+1}^{n} w_{j}\left(\frac{(1-p)^{2}}{1-p_{j}}-\frac{(1+p)^{2}}{1+p_{j}}\right),
$$

where $W_{q}=w_{1}+\cdots+w_{q}$. Using

$$
\frac{(1-p)^{2}}{1-p_{j}}-\frac{(1+p)^{2}}{1+p_{j}} \geq(1-p)^{2}-(1+p)^{2} \text { for } j=q+1, \ldots, n,
$$

we get

$$
\begin{aligned}
\frac{\left(1-p^{2}\right)^{2}}{W_{q}} F_{q}^{\prime}(p) & \geq c p^{c-1}\left(\frac{1-p^{2}}{1-p^{c}}\right)^{2}-4 p+2 p W_{q} \\
& \geq c p^{c-1}\left(\frac{1-p^{2}}{1-p^{c}}\right)^{2}-4 c^{-1} p=G(c, p), \quad \text { say. }
\end{aligned}
$$

Let

$$
E(r, s ; x, y)=\left(\frac{s}{r} \frac{x^{r}-y^{r}}{x^{s}-y^{s}}\right)^{1 /(r-s)}
$$

be the extended mean of order $(r, s)$ of $x, y>0$. Then we have

$$
G(c, p)=4 c^{-1} p^{c-1}(E(2, c ; p, 1))^{4-2 c}-4 c^{-1} p .
$$

Since $1<c<2$ and $E(r, s ; x, y)$ increases with increase in either $r$ or $s$ (see [4]), we obtain

$$
E(2, c ; p, 1) \geq E(2,1 ; p, 1)=\frac{p+1}{2}>p^{1 / 2} .
$$

From (2.11) and (2.12) we conclude that $G(c, p)>0$. This implies that $F_{q}$ is strictly increasing on $\left[p_{q+1}, 1\right)$. Hence, we get

$$
\begin{aligned}
F\left(p_{1}, \ldots, p_{n}\right) & =F_{1}\left(p_{1}\right) \geq F_{1}\left(p_{2}\right)=F_{2}\left(p_{2}\right) \geq F_{2}\left(p_{3}\right) \\
& \geq \cdots \geq F_{n-1}\left(p_{n-1}\right) \geq F_{n-1}\left(p_{n}\right)=\frac{1}{1-p_{n}^{c}}-\frac{1}{1-p_{n}^{2}} \geq 0 .
\end{aligned}
$$

Combining (2.4) and (2.13) it follows that the inequality on the right-hand side of (2.1) is valid. 
4 The inequality of Milne and its converse II

Next, let $\alpha \leq \min (1, a / 2)$. Applying

$$
\min (1, a / 2) \leq \frac{1-p^{a}}{1-p^{2}} \quad(0 \leq p<1)
$$

and the first inequality of (1.1) (with $c_{1}=1$ ) we conclude that the left-hand side of (2.1) holds for all real numbers $p_{j} \in[0,1)(j=1, \ldots, n)$.

It remains to show that the validity of (2.1) implies (2.2). We set $p_{1}=\cdots=p_{n}=p \in$ $(0,1)$. Then the left-hand side of (2.1) leads to

$$
\alpha \leq \frac{1-p^{a}}{1-p^{2}} .
$$

We let $p$ tend to 0 and obtain $\alpha \leq 1$. And, if $p$ tends to 1 , then (2.15) yields $\alpha \leq a / 2$. Let $w=w_{k}$ with $k \in\{1, \ldots, n\}$. We set $p_{j}=0(1 \leq j \leq n ; j \neq k)$ and $p_{k}=p \in(0,1)$. Then the right-hand side of (2.1) is equivalent to

$$
\frac{(1-w+w /(1-p))(1-w+w /(1+p))}{1-w+w /\left(1-p^{b}\right)} \leq \beta .
$$

If $p$ tends to 0 , then $1 \leq \beta$. And, if $p$ tends to 1 , then we get $(1-w / 2) b \leq \beta$.

Remarks 2.2. (i) We define for $b>0$,

$$
H(b)=\max (1,(1-w / 2) b) \sum_{j=1}^{n} \frac{w_{j}}{1-p_{j}^{b}},
$$

where $w_{j}>0(j=1, \ldots, n), w_{1}+\cdots+w_{n}=1, w=\min _{1 \leq j \leq n} w_{j}$, and $p_{j} \in[0,1)(j=$ $1, \ldots, n)$. If $0<b<2 /(2-w)$, then

$$
H^{\prime}(b)=\sum_{j=1}^{n} \frac{w_{j} p_{j}^{b} \log \left(p_{j}\right)}{\left(1-p_{j}^{b}\right)^{2}} \leq 0
$$

And, if $b>2 /(2-w)$, then

$$
H^{\prime}(b)=(1-w / 2) \sum_{j=1}^{n} \frac{w_{j}}{\left(1-p_{j}^{b}\right)^{2}}\left(1-p_{j}^{b}+p_{j}^{b} \log \left(p_{j}^{b}\right)\right) \geq 0 .
$$

This implies that $H$ is decreasing on $(0,2 /(2-w)]$ and increasing on $[2 /(2-w), \infty)$. Hence: if (2.2) holds, then the function

$$
H^{*}(\beta, b)=\beta \sum_{j=1}^{n} \frac{w_{j}}{1-p_{j}^{b}}
$$

satisfies $H^{*}(\beta, b) \geq H^{*}(1,2 /(2-w))$. This means that the expression on the right-hand side of (2.1) attains its smallest value if $\beta=1$ and $b=2 /(2-w)$. Similarly, we obtain: if (2.2) holds, then the expression on the left-hand side of (2.1) attains its largest value if $\alpha=1$ and $a=2$. 
(ii) The upper bounds given in (1.1) with $c_{2}=2-w$ and (2.1) with $\beta=1, b=2 /(2-$ $w)$ cannot be compared. To prove this we set $p_{1}=\cdots=p_{n}=p \in(0,1)$ and denote by $R_{1}(p)$ and $R_{2}(p)$ the expressions on the right-hand side of (1.1) and (2.1), respectively. Then we get

$$
R_{1}(p)=\left(\frac{1}{1-p^{2}}\right)^{c_{2}}, \quad R_{2}(p)=\frac{1}{1-p^{b}} .
$$

First, we show that $R_{1}(p)>R_{2}(p)$ in the neighbourhood of 1 . Let

$$
\Delta(p)=R_{1}(p)-R_{2}(p), \quad \varphi(p)=\left(1-p^{b}\right) \Delta(p) .
$$

Since $c_{2}>1, b>1$ we have

$$
\lim _{p \rightarrow 1} \varphi(p)=\lim _{p \rightarrow 1} \frac{b p^{b-1}}{2 p c_{2}\left(1-p^{2}\right)^{c_{2}-1}}-1=\infty .
$$

This implies that $\varphi$ and $\Delta$ are positive in the neighbourhood of 1 .

Next, we show that $R_{1}(p)<R_{2}(p)$ in the neighbourhood of 0 . Let

$$
\sigma(p)=\Delta\left(p^{1 / 2}\right)
$$

We obtain $\sigma(0)=0$ and since $0<b / 2<1$ we get

$$
\lim _{p \rightarrow 0} \sigma^{\prime}(p)=\lim _{p \rightarrow 0}\left(\frac{c_{2}}{(1-p)^{c_{2}+1}}-\frac{b}{2} p^{b / 2-1} \frac{1}{\left(1-p^{b / 2}\right)^{2}}\right)=-\infty .
$$

This implies that $\sigma$ and $\Delta$ attain negative values in the neighbourhood of 0 .

(iii) The two-parameter mean value family defined in (2.10) has been the subject of intensive research. The main properties are studied in [4-6], where also historical remarks and references can be found.

\section{Matrix inequalities}

We now provide a matrix analogue of Theorem 2.1. The reader who wants to have a proper understanding of the following theorem and its proof needs a general knowledge of matrix theory. We refer to the monographs $[2,3]$.

Theorem 3.1. Let $\alpha, \beta, a>0$, and $b>0$ be real numbers. Further, let $w_{j}(j=1, \ldots, n ; n \geq 2)$ be positive real numbers with $w_{1}+\cdots+w_{n}=1$. The inequalities

$$
\alpha \sum_{j=1}^{n} w_{j}\left(I-P_{j}^{a}\right)^{-1} \leq \sum_{j=1}^{n} w_{j}\left(I-P_{j}\right)^{-1} \sum_{j=1}^{n} w_{j}\left(I+P_{j}\right)^{-1} \leq \beta \sum_{j=1}^{n} w_{j}\left(I-P_{j}^{b}\right)^{-1}
$$

hold for all families of commuting Hermitian matrices $P_{1}, \ldots, P_{n}$, satisfying $0 \leq P_{j}<I$ in the Löwner ordering, if and only if

$$
\alpha \leq \min (1, a / 2), \quad \beta \geq \max \left(1,\left(1-\min _{1 \leq j \leq n} w_{j} / 2\right) b\right) .
$$


Proof. First, we assume that (3.2) is valid. Since the $P_{j}$ commute, there exists a nonsingular matrix $S$ such that $S^{-1} P_{j} S=\operatorname{diag}\left(\ldots, \lambda_{l j}, \ldots\right)$, where $\lambda_{1 j}, \ldots, \lambda_{n j}$ are the eigenvalues of $P_{j}$. By definition of the positive semidefinite ordering (Löwner ordering) it follows that $P_{j}<I$ implies $0 \leq \lambda_{l j}<1$ for $l=1, \ldots, n$. So the expressions given in (3.1) make sense. Denoting by $L, M$, and $R$ the matrices on the left-hand side, in the middle, and on the right-hand side of (3.1), respectively, we get

$$
\begin{gathered}
S^{-1} L S=\operatorname{diag}\left(\ldots, \alpha \sum_{j=1}^{n} \frac{w_{j}}{1-\lambda_{l j}^{a}}, \ldots\right), \quad S^{-1} M S=\operatorname{diag}\left(\ldots, \sum_{j=1}^{n} \frac{w_{j}}{1-\lambda_{l j}} \sum_{j=1}^{n} \frac{w_{j}}{1+\lambda_{l j}}, \ldots\right), \\
S^{-1} R S=\operatorname{diag}\left(\ldots, \beta \sum_{j=1}^{n} \frac{w_{j}}{1-\lambda_{l j}^{b}}, \ldots\right) .
\end{gathered}
$$

Applying Theorem 2.1 we obtain $S^{-1} L S \leq S^{-1} M S \leq S^{-1} R S$, and hence $L \leq M \leq R$.

Next, we suppose that (3.1) holds for all families of commuting Hermitian matrices $P_{1}, \ldots, P_{n}$, satisfying $0 \leq P_{j}<I$. We proceed in analogy with the proof of Theorem 2.1: put $P_{1}=\cdots=P_{n}=\operatorname{diag}(p, \ldots, p)$ with $p \in(0,1)$. Then the left-hand side of (3.1) leads to an inequality for scalar matrices (i.e., multiples of the identity $I$ ), namely,

$$
\alpha \frac{1}{1-p^{a}} I \leq \frac{1}{1-p} I \cdot \frac{1}{1+p} I .
$$

Considering a pair of corresponding diagonal entries we conclude that this inequality is equivalent to (2.15). Tending with $p$ to 0 and 1 , respectively, we get $\alpha \leq \min (1, a / 2)$. Next, let $w=w_{k}$, where $k \in\{1, \ldots, n\}$. We set $P_{j}=0$ for $j \neq k$ and $P_{k}=p I$. Then the right-hand side of (3.1) yields

$$
((1-w) I+(w /(1-p)) I) \cdot((1-w) I+(w /(1+p)) I) \leq \beta\left((1-w) I+\left(w /\left(1-p^{b}\right)\right) I\right)
$$

Again, this is an inequality for scalar matrices and it suffices to consider diagonal entries. This leads to (2.16). We let $p$ tend to 0 and 1, respectively, and obtain the second of the inequalities (3.2).

\section{References}

[1] H. Alzer and A. Kovačec, The inequality of Milne and its converse, Journal of Inequalities and Applications 7 (2002), no. 4, 603-611.

[2] R. A. Horn and C. R. Johnson, Matrix Analysis, Cambridge University Press, Cambridge, 1985.

[3] _ Topics in Matrix Analysis, Cambridge University Press, Cambridge, 1991.

[4] E. B. Leach and M. C. Sholander, Extended mean values, The American Mathematical Monthly 85 (1978), no. 2, 84-90.

[5] Extended mean values. II, Journal of Mathematical Analysis and Applications 92 (1983), no. 1, 207-223.

[6] - Multi-variable extended mean values, Journal of Mathematical Analysis and Applications 104 (1984), no. 2, 390-407. 
[7] E. A. Milne, Note on Rosseland's integral for the stellar absorption coefficient, Monthly Notices of the Royal Astronomical Society 85 (1925), 979-984.

[8] C. R. Rao, Statistical proofs of some matrix inequalities, Linear Algebra and its Applications 321 (2000), no. 1-3, 307-320.

Horst Alzer: Morsbacher Street 10, 51545 Waldbröl, Germany

E-mail address: alzerhorst@freenet.de

Alexander Kovačec: Departamento de Matemática, Universidade de Coimbra, 3001-454 Coimbra, Portugal

E-mail address: kovacec@mat.uc.pt 